ARTICLE

Received 19 Feb 2014 | Accepted 30 Jun 2014 | Published 31 Jul $2014 \quad$ DOl: 10.1038/ncomms5561

\title{
Sustained GSK3 activity markedly facilitates nerve regeneration
}

\author{
Philipp Gobrecht ${ }^{1}$, Marco Leibinger ${ }^{1}$, Anastasia Andreadaki ${ }^{1} \&$ Dietmar Fischer $^{1}$
}

Promotion of axonal growth of injured DRG neurons improves the functional recovery associated with peripheral nerve regeneration. Both isoforms of glycogen synthase kinase 3 (GSK3; $\alpha$ and $\beta$ ) are phosphorylated and inactivated via phosphatidylinositide 3-kinase $(\mathrm{PISK}) / \mathrm{AKT}$ signalling upon sciatic nerve crush (SNC). However, the role of GSK3 phosphorylation in this context is highly controversial. Here we use knock-in mice expressing GSK3 isoforms resistant to inhibitory PI3K/AKT phosphorylation, and unexpectedly find markedly accelerated axon growth of DRG neurons in culture and in vivo after SNC compared with controls. Moreover, this enhanced regeneration strikingly accelerates functional recovery after SNC. These effects are GSK3 activity dependent and associated with elevated MAP1B phosphorylation. Altogether, our data suggest that PI3K/AKT-mediated inhibitory phosphorylation of GSK3 limits the regenerative outcome after peripheral nerve injury. Therefore, suppression of this internal 'regenerative break' may potentially provide a new perspective for the clinical treatment of nerve injuries.

\footnotetext{
${ }^{1}$ Division of Experimental Neurology, Department of Neurology, Heinrich Heine University of Düsseldorf, Merowingerplatz 1a, 40225 Düsseldorf, Germany Correspondence and requests for materials should be addressed to D.F. (email: dietmar.fischer@uni-duesseldorf.de).
} 
n contrast to the central nervous system (CNS), injured axons in the peripheral nervous system normally regenerate and a small percentage even reconnects to their appropriate target. This successful regeneration is associated with significant, though often incomplete functional restoration ${ }^{1,2}$. Measures increasing the growth rate of regenerating axons reportedly improve the functional outcome after peripheral nerve lesion and are therefore from a clinical point of view desirable ${ }^{3}$.

Emerging evidence points to a relevant role for glycogen synthase kinase 3 (GSK3) in peripheral nerve regeneration ${ }^{4-9}$. Both isoforms, GSK3 $\alpha$ and GSK3 $\beta$, are serine/threonine kinases sharing across species a homology of $85 \%$ (refs 7,10 ). They both have a high basal activity in resting cells, but are inactivated by AKT-induced phosphorylation of serine 21 (GSK3 $\alpha$ ) and serine 9 (GSK3 $\beta)$, respectively $4,11,12$. However, the effect attributed to GSK3 phosphorylation with regard to axon growth as well as myelin-inhibition remains controversial. Pharmacological inhibition of GSK3 reportedly improves axon regeneration in vivo by desensitizing axons towards myelin and other CNS inhibitors ${ }^{5}$, suggesting that GSK3 $\beta$ phosphorylation facilitates axon regeneration. On the other hand, other data demonstrate that GSK3 phosphorylation is involved in inhibitory processes and therefore limits the regenerative outcome ${ }^{6}$. Similarly, reports regarding GSK3's role in axon growth initiation are also inconsistent. Previous studies provided evidence that inactivation of GSK3 compromises axon growth ${ }^{13-15}$ and that active GSK3 is favourable for axon growth during development as it phosphorylates microtubule-associated protein 1B (MAP1B), which shifts microtubules in a dynamic state that promotes axon elongation ${ }^{14,16}$. On the other hand others suggest that spatially localized inactivation of GSK3 at the distal axon is required for efficient axon elongation of DRG neurons ${ }^{4}$. In addition, a recent study reported that phosphatidylinositide 3-kinase (PI3K)/AKT-mediated phosphorylation of GSK3 and subsequent SMAD1 expression regulates peripheral nerve regeneration, thereby suggesting that inactivation of GSK3 by AKT is essential for switching adult DRG neurons into a regenerative state . $^{8}$

Most studies regarding the role of GSK3 in axon regeneration so far rely mainly on pharmacological manipulation of GSK3 activity. Therefore, inconsistent results may potentially be attributable to off-target effects and the use of different inhibitors. In addition, exogenous overexpression of GSK3 might induce unphysiological high levels of GSK3. To avoid these potential complications, we took advantage of GSK $3 \alpha /$ GSK $3 \beta$ double knock-in mice (GSK3 ${ }^{\mathrm{S} / \mathrm{A}}$ ) to clarify the role of GSK3 phosphorylation in peripheral nerve regeneration. In these mice, serine 21 of GSK $3 \alpha$ and serine 9 of GSK3 $\beta$ are substituted by alanine, preventing GSK3 phosphorylation by $\mathrm{AKT}^{17}$, thereby rendering GSK3 constitutively active without changing its physiological expression level. The current study demonstrates that both GSK3 isoforms are phosphorylated PI3K/AKT pathway dependently in adult wild-type (wt) DRG neurons after axotomy. We found that global prevention of Ser21/Ser9 GSK3 phosphorylation markedly enhances axon growth of cultured DRG neurons kinase activity dependently and leads to accelerated sciatic nerve regeneration and functional recovery.

\section{Results}

Axotomy induces serine phosphorylation of GSK3 $\alpha$ and GSK3及. GSK3 is increasingly emerging as a pivotal player in peripheral nerve regeneration, but the relevance of its activity/ phosphorylation with regard to axon growth induction remains highly controversial. To analyze the phosphorylation status of GSK3 in injured dorsal root ganglion (DRG) neurons, adult wildtype (wt) mice were subjected to sciatic nerve crush (SNC). As determined by immunohistochemistry, lumbar (L3, L4) DRG neurons of untreated control mice, whose axons project into the sciatic nerve ${ }^{18}$ revealed very little phospho-S21-GSK3 $\alpha$ (pGSK3 $\alpha$ ) and phospho-S9-GSK3 $\beta$ (pGSK3 $\beta$ ) staining (Fig. 1a). In comparison, levels of both pGSK $3 \alpha$ and pGSK3 $\beta$ were markedly increased 5 days after SNC (Fig. 1a). The specificity of the applied antibodies was verified by the absence of any signal in DRG from homozygous GSK3 $\alpha^{\text {Ser21Ala }} / G S K 3 \beta^{\text {Ser9Ala }}$ double knock-in $\left(\mathrm{GSK}^{\mathrm{S} / \mathrm{A}}\right)$ mice, which are incapable of phosphorylating GSK3 at serines 21 and 9, respectively. This increase in pGSK $3 \alpha$ and pGSK3 $\beta$ levels was confirmed by Western-blot analysis of L3/L4 DRG lysates isolated 5 days after SNC in comparison to untreated controls. Again lysates from GSK3 ${ }^{\mathrm{S} / \mathrm{A}}$ mice were used as specificity controls and expectedly showed no signal. Elevated GSK3 phosphorylation compared with uninjured controls was not only detected in neuronal cell bodies, but also in injured sciatic nerve tissue (Fig. 1b, Supplementary Fig. 1). In addition, GSK3 phosphorylation was induced in DRG neurons after dissociation-induced axotomy (Fig. 1c), as the numbers of pGSK $3 \alpha$ - and pGSK3 $\beta$-positive cells were significantly increased at 3 days compared with $2 \mathrm{~h}$ after culture preparation (Fig. 1c, Fig. 2b). Therefore, phosphorylation of GSK3 at serines 21 and 9, respectively, is markedly induced in adult DRG neurons upon axotomy.

GSK3 phosphorylation depends on PI3K/AKT signalling. Next we addressed the question, which signalling pathway(s) might mediate this axotomy-induced GSK3 phosphorylation. To this end, the number of pGSK $3 \alpha$ - and pGSK3 $\beta$-positive DRG neurons cultured in the presence of various signalling pathway inhibitors was determined (Fig. 2). Expectedly, axotomy-induced GSK3 $\alpha$ and GSK3 $\beta$-phosphorylation was abrogated with the PI3K/AKT inhibitor (LY294002), but not with inhibitors of mammalian Target of Rapamycin (mTOR) (rapamycin), the JAK/STAT3(AG490) or the MAPK/ERK-signalling pathways (PD98059 and U0126) (Fig. 2a-c). Lithium, which induces inhibitory GSK3 phosphorylation via AKT activation and protein phosphatase-1 suppression $^{19}$ served as positive control, expectedly further increasing GSK3 phosphorylation (Fig. 2a-c).

Prevention of GSK3 phosphorylation promotes axonal growth. Previous studies suggested a requirement of GSK3 serine phosphorylation for the transformation of DRG neurons into a regenerative state ${ }^{4,8}$. We therefore compared the axon growth capacity of DRG neurons isolated from transgenic GSK3 ${ }^{\mathrm{S}}{ }_{\mathrm{A}}^{\mathrm{A}}$ with wt mice in vitro. Surprisingly, GSK3 ${ }^{\text {S/A }}$ DRG neurons showed markedly increased axonal growth in comparison to neurons from wt animals (Fig. 3a,b). Interestingly, growth induction was comparable for neurons from homo- and heterozygous knock-in mice. Therefore, the observed growth promotion seemed to be mediated by sustained GSK3 activity rather than the complete absence of phosphorylated GSK3. Survival of cultured DRG neurons was similar in all experimental conditions, indicating that GSK3 ${ }^{\mathrm{S} / \mathrm{A}}$-mediated effects were restricted to axon growth (Supplementary Fig. 2).

In addition, we tested the effect of $\mathrm{GSK} 3^{\mathrm{S} / \mathrm{A}}$ on conditioning lesion-induced axon growth, employing a replating method described previously ${ }^{8}$. Therefore DRG neurons were cultured for 3 days and replated afterwards overnight (Fig. 3c). Similarly, axon growth from GSK3 ${ }^{\text {S/A }}$ neurons was significantly stronger compared with neurons from wt animals, providing further evidence that GSK3 phosphorylation is not required for axon growth stimulation.

GSK3 $3^{\text {S/A }}$-stimulated axon growth depends on kinase activity. To test our hypothesis that GSK3 ${ }^{\mathrm{S} / \mathrm{A}}$-mediated axon growth 
a wt
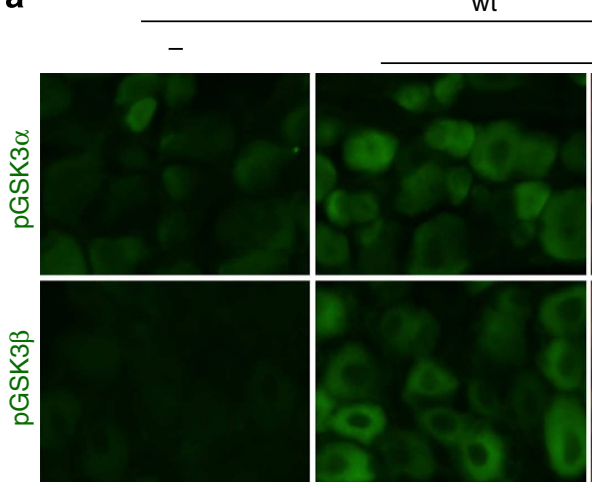

$5 \mathrm{dSNC}$

GSK3 $^{\mathrm{S} / \mathrm{A}}$
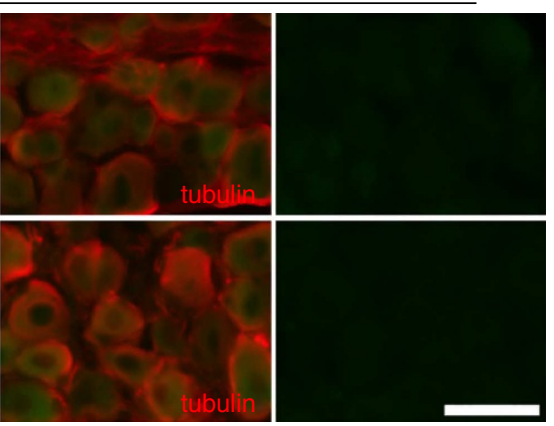

b
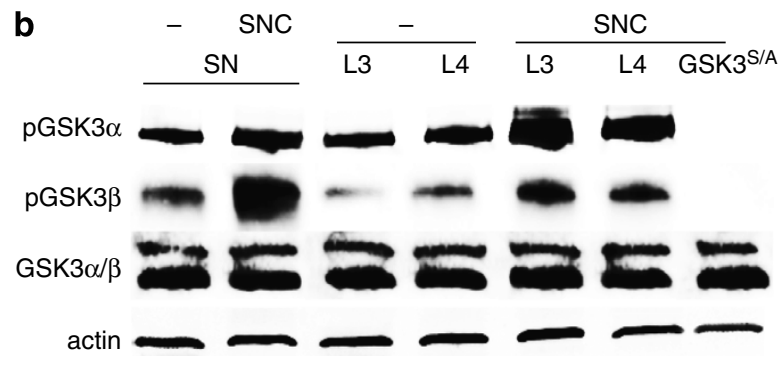

C
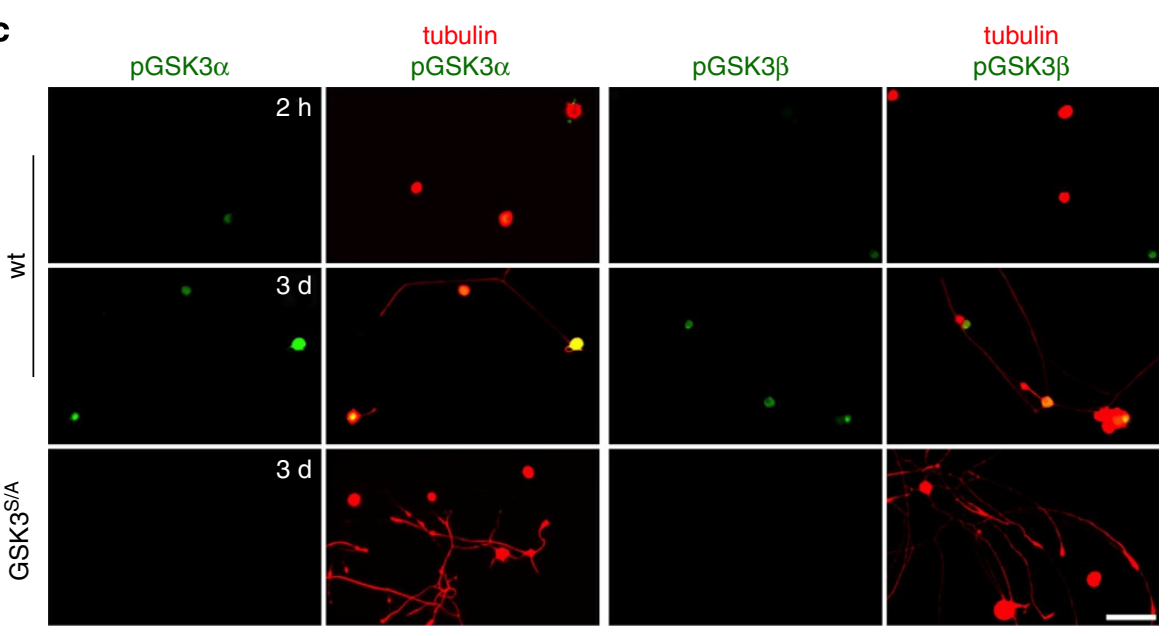

Figure 1 | Sciatic nerve injury induces GSK3 $\alpha$ and GSK3 $\beta$ phosphorylation. (a) Immunohistochemical detection of phospho-S21-GSK3 $\alpha$ (pGSK3 $\alpha$ ) and phospho-S9-GSK3 $\beta$ (pGSK3 $\beta$ ) (green) on DRG sections from wild-type (wt) and GSK3 double knock-in mice (GSK3 ${ }^{S / A}$ ). Animals received either no treatment $(-)$ or a sciatic nerve crush (SNC) 5 days prior to tissue preparation. Both GSK3 isoforms were phosphorylated upon SNC in DRG neurons as detected by $\beta$ III tubulin staining (red). Scale bar, $50 \mu \mathrm{m}$. (b) Western-blot analysis detecting pGSK3 $\alpha$, pGSK3 $\beta$ as well as total GSK3 $\alpha / \beta$ in lysates prepared from lumbar DRG $(L 3, L 4)$ and sciatic nerves $(S N)$ isolated from either untreated $(-)$ or from animals subjected to SNC 5 days prior to tissue isolation. In contrast to total GSK3, both phosphorylated isoforms were upregulated in DRG and proximal sciatic nerve stumps upon injury. Lysates from GSK3 $3 / A$ animals verified the specificity of the antibodies. Actin served as additional loading control. (c) Immunocytochemical staining of dissociated adult mouse DRG cultures derived from either wt or GSK3 $3 / A$ transgenic mice using antibodies against phospho-S21-GSK3 $\alpha$ ( $\alpha$ ) or phospho-S9-GSK3 $\beta$ ( $\beta$ ) (green) and $\beta I I I-t u b u l i n$ (red) to detect DRG neurons. Cultures were incubated either for $2 \mathrm{~h}$ or 3 days as indicated. GSK3 phosphorylation was induced in dissociated adult DRG neurons. Scale bar, $50 \mu \mathrm{m}$.

promotion depends on sustained kinase activity rather than the ablation of phosphorylated GSK3, cultured DRG neurons isolated from either GSK3 ${ }^{S / A}$ or wt mice were exposed to the GSK3 inhibitors lithium and SB415286 (SB), respectively (Fig. 4). In contrast to lithium, SB directly blocks the substrate-binding site of GSK3 to reduce its activity instead of relying on inhibitory phosphorylation of serines $21 / 9$ (ref. 20). In contrast to lithium, which did not affect the increased axon growth of GSK3 ${ }^{\text {S/A }}$ DRG neurons, axon growth was significantly reduced by SB (Fig. $4 \mathrm{~b}$ ). These data suggest a kinase activity-dependent mechanism of $\mathrm{GSK}^{\mathrm{S} / \mathrm{A}}$ for axon growth promotion.
As GSK3 ${ }^{\text {S/A }}$ might nevertheless be inactivated by an unknown mechanism, we also examined the phosphorylation levels of MAP1B, a well-known substrate of GSK3, which does not require prior priming ${ }^{16}$. Wt DRG cultures contained only very few neurons with pMAP1B-positive axons. In contrast, pMAP1B was detected in almost all axons of GSK3 ${ }^{\mathrm{S} / \mathrm{A}}$ neurons, indicating robust GSK activity (Fig. 4d). Accordingly, MAP1B phosphorylation was blocked in the presence of the GSK3 inhibitor SB, but not lithium.

GSK3 activity reportedly modulates the stability of microtubules, thereby affecting axonal growth ${ }^{21-23}$. We therefore 
a

$2 \mathrm{~h}$

3 days

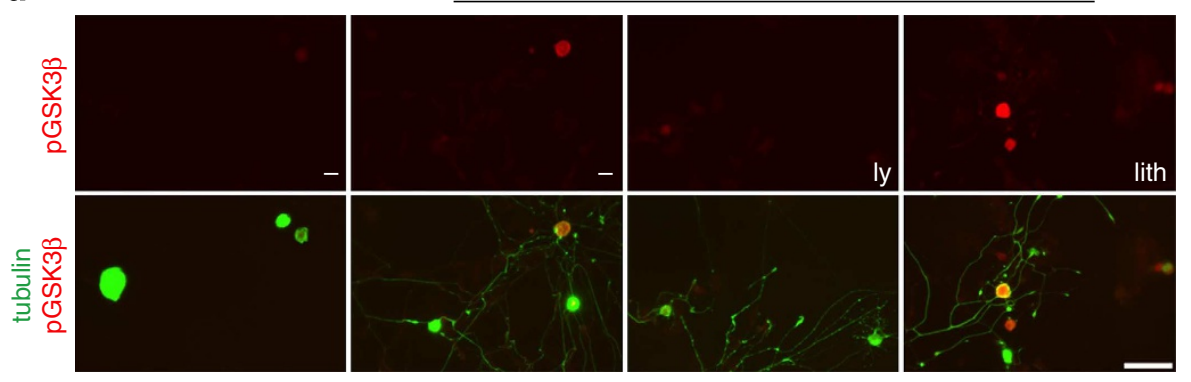

b
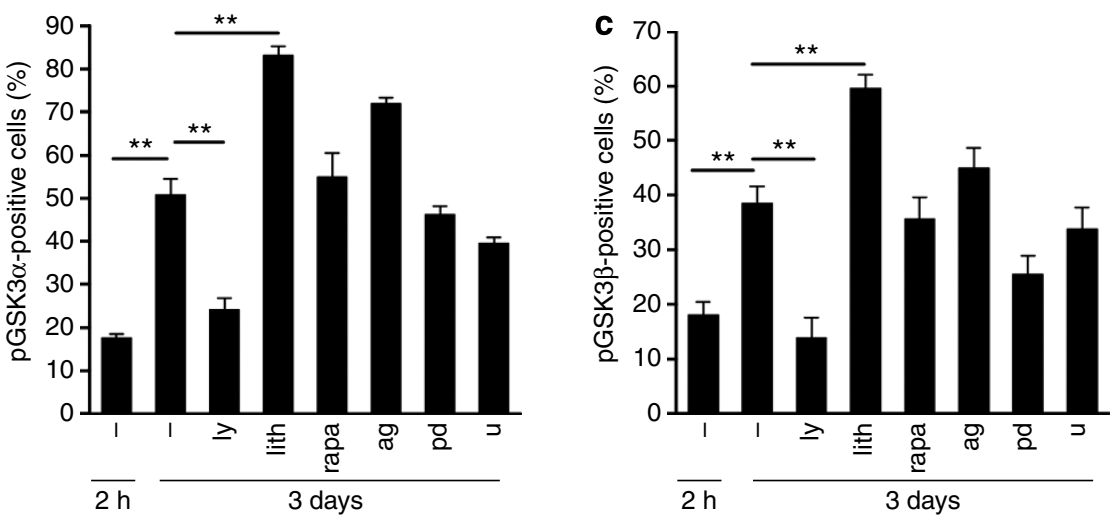

Figure 2 | Axotomy-induced GSK3 phosphorylation is PI3K/AKT dependent. (a) Dissociated adult DRG cultures were immunocytochemically stained for phospho-S21-GSK3 $\beta$ (red) and $\beta$ III-tubulin (green) after $2 \mathrm{~h}$ or 3 days in culture. DRG cultures had been treated with vehicle $(-$ ), PI3K inhibitor LY294002 $(\mathrm{ly}, 1 \mu \mathrm{M}$ ) or GSK3 inhibitor lithium (lith, $3 \mathrm{mM}$ ). Scale bar, $100 \mu \mathrm{m}$. (b) Quantification of pGSK3 $\alpha$ positive DRG neurons in experimental groups as described in a and additional treatments with the mTOR inhibitor rapamycin (rapa, $10 \mathrm{nM}$ ), the JAK inhibitor AG490 (ag, $5 \mu \mathrm{M}$ ) and the ERK inhibitors PD98059 (pd, $5 \mu \mathrm{M})$ and U0126 $(\mathrm{u}, 5 \mu \mathrm{M})$. (c) Quantification of pGSK3 $\beta$ positive DRG neurons in cultures as described in b. Experiments shown in a-c were performed three times each. The error bars represent the standard error of the mean. Statistical significance was determined with ANOVA and the post-hoc Holm Sidac test. Treatment effects: ${ }^{\star \star} P \leq 0.001$.

reasoned that axonal growth of GSK3 ${ }^{\mathrm{S} / \mathrm{A}}$ neurons might be more susceptible to microtubules-disrupting agents. To address this possibiity we exposed DRG cultures to different concentrations of nocodazole, which induces the depolymerization of microtubules. Nocodazol concentrations not affecting axonal growth of wt neurons (Fig. 4a,b) significantly reduced growth of GSK3 3 /A neurons to comparable levels as in the wt and thereby blocking the growth-promoting $\mathrm{GSK}^{\mathrm{S} / \mathrm{A}}$ effect. In conclusion, the overall beneficial effect of constitutively active GSK3 may, at least partially, be mediated by the modulation of microtubules dynamics.

Effects of GSK3 phosphorylation on gene expression. Transformation of injured DRG neurons into a regenerative state and associated PI3K/AKT-induced GAP43 expression has recently been reported to depend on inhibitory GSK3 serine phosphorylation ${ }^{8}$. We therefore investigated whether the induction of regeneration-associated-genes, such as GAP43 and SPRR1a might be affected in GSK3 ${ }^{\text {S/A }}$ mice. As measured by quantitative real-time PCR, expression of both genes was strongly induced 5 days after SNC in wt as well as in GSK3 ${ }^{\mathrm{S} / \mathrm{A}}$ mice (Fig. 5a,b). Consistent with our functional data, expression of GAP43 and SPRR1a was not reduced, but rather slightly elevated in $\mathrm{GSK}^{\mathrm{S} / \mathrm{A}}$ compared with wt mice. Similar GAP43-protein expression of equally treated samples from GSK3 mutant and wt animals was confirmed by Western-blot analysis (Fig. 5c) and immunocytochemistry on cultured DRG neurons (Fig. 5d).

Saijilafu et al. ${ }^{8}$ also suggested that PI3K/AKT/GSK3 regulates axon regeneration by the induction of SMAD1 expression. We therefore analyzed the expression of this transcription factor upon SNC in GSK3 ${ }^{\mathrm{S} / \mathrm{A}}$ animals. Consistent with Saijilafu et al. ${ }^{8}$, we found that the overall expression of SMAD1 was strongly reduced in $\mathrm{GSK} 3{ }^{\mathrm{S} / \mathrm{A}}$ mice compared with wt animals (Fig. $5 \mathrm{c}$ ), verifying that the cellular presence of SMAD1 depends on GSK3 activity. However, despite the strongly reduced overall expression of SMAD1 in GSK3 ${ }^{\mathrm{S} / \mathrm{A}}$ mice its expression was still increased by SNC, suggesting that additional mechanisms are involved in this process. Moreover, these data demonstrate that elevation of SMAD1 expression above basal levels is not essentially required for axon regeneration.

Sustained GSK3 activity facilitates axonal regeneration in vivo. As we observed markedly increased axonal growth of cultured GSK3 ${ }^{\text {S/A }}$ DRG neurons, we next investigated the impact of $\mathrm{GSK}^{\mathrm{S} / \mathrm{A}}$ on sciatic nerve regeneration in vivo. To this end, the number of SCG10-positive, regenerating axons was quantified at varying distances beyond the lesion site at 3 days after SNC. Regeneration was markedly increased particularly at longer distances in GSK3 ${ }^{\mathrm{S} / \mathrm{A}}$ mice compared with wt controls (Fig. $6 \mathrm{a}-\mathrm{d}, \mathrm{f}$ ). Beyond $3 \mathrm{~mm}$ from the lesion site, axons were only detected in $\mathrm{GSK}^{\mathrm{S} / \mathrm{A}}$, but not wt animals (Fig. 6a-d) indicating accelerated axonal growth. These findings were confirmed by neurofilament staining of respective sciatic nerve cross-sections at $3 \mathrm{~mm}$ past the crush site 3 days after injury (Fig. 6f). In addition, regenerating axons of GSK3 ${ }^{\mathrm{S} / \mathrm{A}}$ mice showed a higher staining intensity for pMAP1B compared with wt animals (Fig. 6e), indicating an elevated GSK3 activity in regenerating axons of GSK3 ${ }^{\mathrm{S} / \mathrm{A}}$ animals.

Functional recovery is accelerated in $\mathrm{GSK} 3^{\mathrm{S} / \mathrm{A}}$ mice after SNC. Motor and sensory recovery upon SNC are commonly assessed 

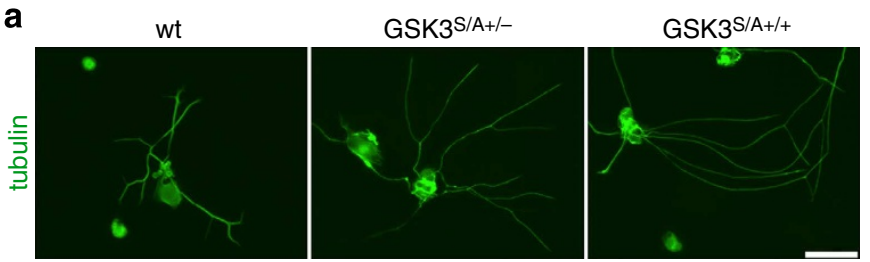

b

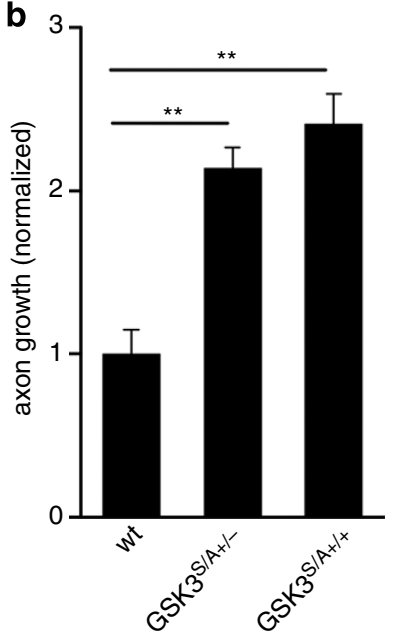

C

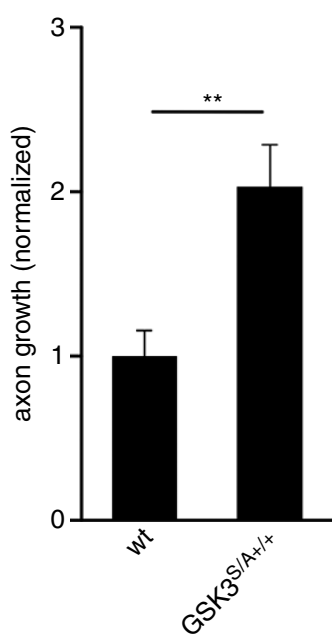

Figure 3 | Impediment of GSK3 phosphorylation promotes axonal

growth. (a) Dissociated DRG neurons isolated from either wild-type (wt), heterozygous (GSK3 $\mathrm{S} / \mathrm{A}+/-$ ) or homozygous $\left(\mathrm{GSK} 3^{\mathrm{S} / \mathrm{A}+/+}\right.$ )

GSK3 $\alpha^{\text {S21A }} /$ GSK3 $\beta^{\text {S9A }}$ double knock-in mice were stained with $\beta$ III-tubulin after 2 days in culture. Scale bar, $100 \mu \mathrm{m}$. (b) Quantification of DRG axon growth of cultures depicted in $\mathbf{a}$. Data were normalized from three independent experiments to the vehicle-treated control group with an average axon length of $193 \mu \mathrm{m}$ per DRG neuron. Homo and heterozygous GSK3 ${ }^{\text {S/A }}$ neurons show significantly increased axonal growth compared with wt controls. (c) Quantification of axon growth of adult DRG neurons from wt and double knock-in mice $20 \mathrm{~h}$ after replating. Data from two independent experiments were normalized to the control group with an average axon length of $21 \mu \mathrm{m}$ per DRG neuron. The error bars in $\mathbf{b}$ and $\mathbf{c}$ represent the standard error of the mean. Statistical significance was determined with ANOVA and the post-hoc Holm Sidac test. Treatment effects: ${ }^{\star \star} P \leq 0.001$.

using the toe spreading and the von Frey tests, respectively ${ }^{24,25}$. Therefore, we determined the static sciatic index (SSI) in GSK3 ${ }^{\mathrm{S} / \mathrm{A}}$ compared with wt mice ${ }^{26}$. No statistical differences in baseline SSI and SSI at 1 and 4 days after SNC was detected between uninjured mice (Fig. 7a,b). However, the SSI was significantly higher at 7-21 days after SNC in GSK3 ${ }^{\text {S/A }}$ compared with wt mice, indicating improved motor function recovery (Fig. 7a,b). The re-establishment of neuromuscular junctions 7 days after $\mathrm{SCN}$ in $\mathrm{GSK} 3^{\mathrm{S} / \mathrm{A}}$ mice was confirmed by neurofilament and $\alpha$-bungarotoxin co-stainings of extensor hallucis longus (EHL) muscles. Re-established neuromuscular junctions were only found in GSK3 $3^{\mathrm{S} / \mathrm{A}}$ but not in wt muscles at this time point (Fig. 7c).

Sensory recovery was measured using the von Frey test that determines the thickness of a filament required to induce a hind paw withdrawal response ${ }^{25}$. Uninjured wt and $\mathrm{GSK}^{\mathrm{S} / \mathrm{A}}$ animals showed comparable baseline responses (Fig. $7 \mathrm{~d}$ ). The required force markedly increased to a similar extent in both groups at 1 day after SNC, indicating a dramatic loss of touch sensation after SNC. Animals started to recover touch sensation 7 days after injury and continued over a time period of 21 days with GSK3 ${ }^{\text {S/A }}$ animals improving significantly faster than wt animals at all time points analyzed (Fig. 7d). In summary, these data demonstrate that increased axon growth was associated with accelerated functional recovery upon injury.

\section{Discussion}

The current study investigated the role of AKT-mediated inhibitory GSK3 phosphorylation in adult peripheral nerve regeneration. Surprisingly, maintained GSK3 activity in $\mathrm{GSK}^{\mathrm{S} / \mathrm{A}}$ neurons markedly promoted axon regeneration and accelerated functional recovery after peripheral nerve injury in adult mice. These findings reveal an unexpected and new role of GSK3 in this context and are potentially meaningful for the development of novel therapies for peripheral nerve injury.

The essential role of the PI3K/AKT signalling pathway in stimulating axon regenerative processes in the adult central and peripheral nervous systems is well documented ${ }^{8,27-30}$. Activated AKT phosphorylates GSK3 $\alpha$ at serine 21 (pGSK3 $\alpha$ ) and GSK3 $\beta$ at serine 9 (pGSK3 $\beta$ ) and thereby inhibits their respective kinase activity $7,31,32$. Consistent with elevated PI3/AKT activity in DRG neurons upon peripheral nerve injury ${ }^{8}$, the current study found a robust increase of pGSK $3 \alpha$ and pGSK3 $\beta$ levels in DRG neurons and the proximal sciatic nerve upon SNC. In addition, DRG neurons also increased pGSK3 levels after a few hours in culture, most likely due to the axotomy associated with tissue dissociation. Inhibition of GSK3 phosphorylation in the presence of a PI3K inhibitor confirmed the dependence of this process on the PI3K/ AKT signalling pathway.

A previous report suggested that inhibitory phosphorylation of GSK3s is essentially required for transforming DRG neurons into a regenerative state and therefore for axon regeneration upon injury in general ${ }^{8}$. This notion is mainly based on observations that the inhibitory effect of a PI3K inhibitor on axon growth of cultured DRG neurons was rescued in the presence of pharmacological GSK3 inhibitors ${ }^{8}$. However, some of these inhibitors show low selectivity and high $\mathrm{IC}_{50}$ values potentially affecting other structurally similar kinases ${ }^{33,34}$. Complementary supportive experiments testing whether genetically modified GSK3 without the inhibitory GSK3-phosphorylation site would compromise axon growth were not performed. To address this point in our study, we used well-established GSK3 double knockin mice, which are globally resistant to AKT-mediated GSK3 phosphorylation at serine 21/9 (refs 17,35). Instead of the expected decrease, axon regeneration in cell culture and in vivo was markedly improved in GSK3 $3^{\text {S/A }}$ neurons compared with controls even when the neurons were primed after replating. Moreover, induction of the regeneration-associated genes GAP43 and SPRR1A upon SNC was not compromised in GSK3 ${ }^{\mathrm{S} / \mathrm{A}}$ animals, but rather slightly increased. Therefore, our data suggest that (1) GSK3 phosphorylation is not essentially required for the induction of GAP43 expression or the transformation of adult DRG neurons into a regenerative state and (2) PI3K/AKT-mediated GSK3 phosphorylation partially limits peripheral nerve regeneration.

In addition, our data indicate that the observed axon growthpromoting effect depends on an overall higher kinase activity in non-phosphorylatable GSK3 ${ }^{\mathrm{S} / \mathrm{A}}$ neurons. The presence of SB415286, a competitive inhibitor directly blocking the substrate-binding site of GSK3 (ref. 20), specifically abrogated increased axonal growth, while lithium, a non-competitive inhibitor impeding GSK3 activity via S21/9 phosphorylation, showed no effect on GSK3 ${ }^{S / A}$ neurons. Together with the observation that direct phosphorylation of MAP1B at Th1265 by GSK3, which does not require prior priming by other kinases $16,22,36$, was significantly elevated in neurons of double knock-in mice, these data reflect an overall higher kinase activity of $\mathrm{GSK} 3^{\mathrm{S} / \mathrm{A}}$ compared with controls. Moreover, these data 
a
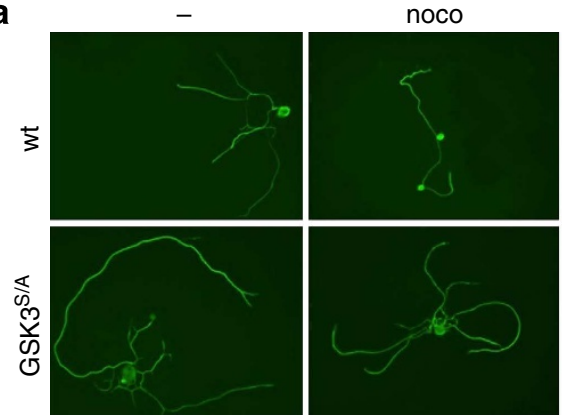

b

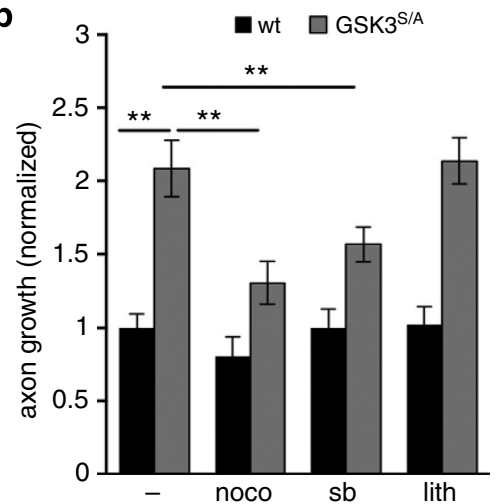

$\mathrm{sb}$
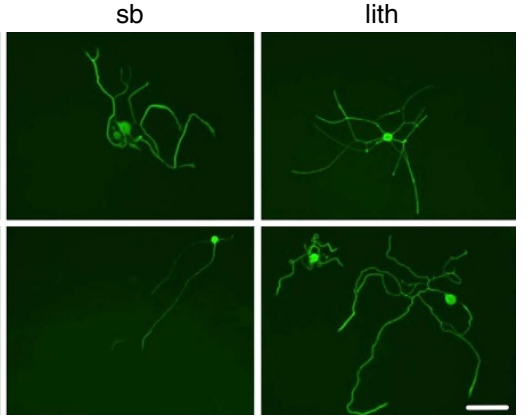

\section{C}

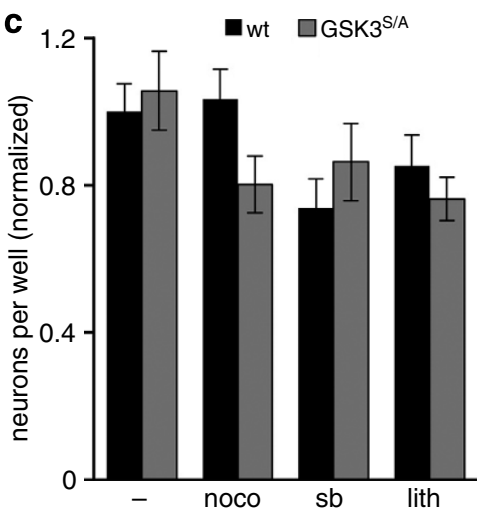

\section{d}

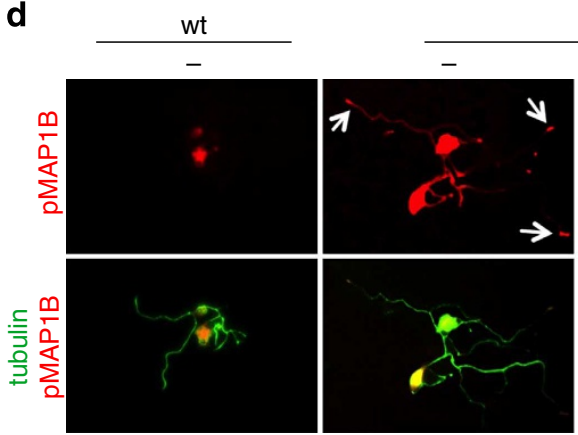

GSK3 $^{\mathrm{S} / \mathrm{A}}$

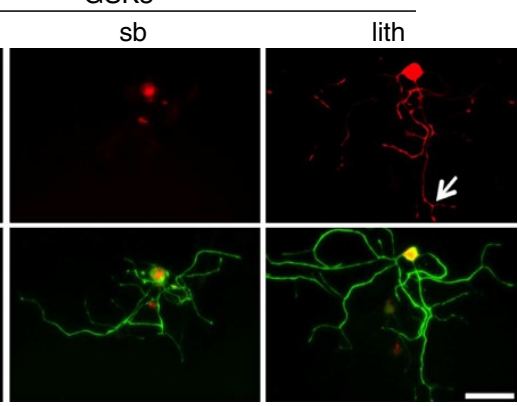

Figure 4 | Axon growth stimulation depends on GSK3 ${ }^{\text {S/A }}$ kinase activity. (a) $\beta$ III-tubulin-positive mouse DRG neurons of either wild-type (wt) or GSK3 $5 / A$ mice were exposed to either vehicle ( - ), nocodazole (noco, $10 \mathrm{nM}$ ), SB415286 (sb, $5 \mu \mathrm{M}$ ) or lithium chloride (lith, $3 \mathrm{mM}$ ) for 2 days in culture. Scale bar, $100 \mu \mathrm{m}$. (b) Quantification of DRG axon growth in cultures depicted in a. Values from three independent experiments were normalized to vehicle-treated control groups with an average axon length of $143 \mu \mathrm{m}$ per DRG neuron. Axon growth stimulation by GSK3 ${ }^{\mathrm{S} / \mathrm{A}}$ neurons is blocked by nocodazole and SB415286, but not lithium indicating kinase activity dependence. The error bars represent the standard error of the mean. Statistical significance was determined with ANOVA and the post-hoc Holm Sidac test. Treatment effects: ${ }^{\star \star} P \leq 0.001$. (c) Quantification of the number of DRG neurons for each experimental group shown in $\mathbf{a}$, demonstrating similar numbers per well. Data of three independent experiments were normalized to the control group (78 DRG neurons per well). The error bars represent the standard error of the mean. (d) Dissociated adult mouse DRG cultures from either wt or GSK3 ${ }^{S / A}$ mice were exposed either to vehicle, lithium (lith, 3 mM) or SB415286 (sb, $5 \mu$ M) for 3 days and stained with antibodies against phospho-MAP1B (pMAP1B, red) $\beta$ III-tubulin (green). Arrows indicate axons. GSK3 ${ }^{S / A}$ neurons show stronger pMAP1B staining than controls. Elevation of pMAP1B levels in GSK3 ${ }^{S / A}$ neurons is blocked in the presence of SB415286, but not lithium, verifying the dependence on GSK3 activity. Scale bar, $50 \mu \mathrm{m}$.

exclude the inhibition of the GSK3 kinase activity by another, unknown compensatory mechanism in GSK3 ${ }^{S / A}$ mice. This idea is also supported by the strong reduction of SMAD1 expression in

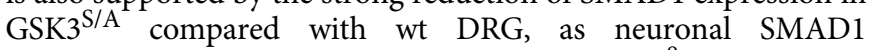
expression levels are regulated by GSK3 activity ${ }^{8}$. However, as axon regeneration was promoted in GSK3 ${ }^{\mathrm{S} / \mathrm{A}}$ mice, these data demonstrate that elevation of SMAD1 expression above basal levels in wt animals is not required for the transformation of DRG neurons into a regenerative state, without excluding the possibility that basal SMAD1 levels are essential for axon growth ${ }^{37}$. Interestingly, despite the overall strong reduction of SMAD1 expression in GSK3 $3^{\mathrm{S} / \mathrm{A}}$ mice, expression of this transcription factor was still increased after SNC in these animals, demonstrating GSK3 phosphorylation-independent upregulation. Altogether these data do not support the concept that PI3K-GSK3 signalling mainly regulates axon regeneration by inducing the expression of Smad1 as recently stated by others ${ }^{8}$.

To our knowledge this is the first report showing that maintenance of GSK3 activity facilitates axon regeneration in the adult nervous system. In the developing nervous system, GSK3 regulates the activity of several microtubule associated proteins (MAPs) $4,13,21-23,38,39$ and thereby controls directed growth cone advancement and guidance. One of these proteins is MAP1B, which is required for axon growth ${ }^{40}$. MAP1B exists in at least two different modes of phosphorylation ${ }^{41}$, with mode II phosphorylation being mediated mainly by casein kinase II 

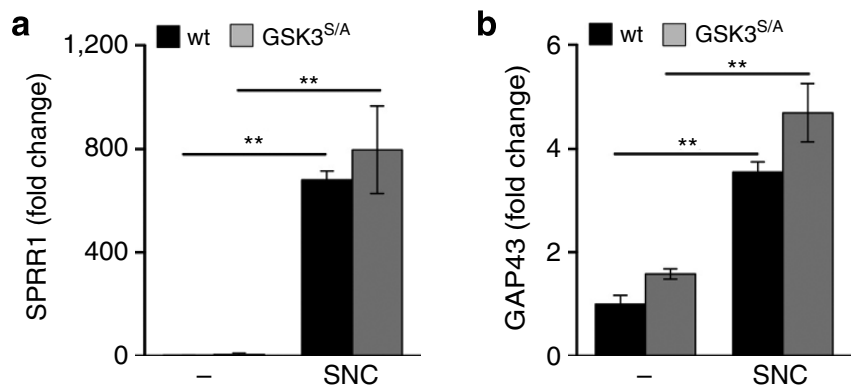

C
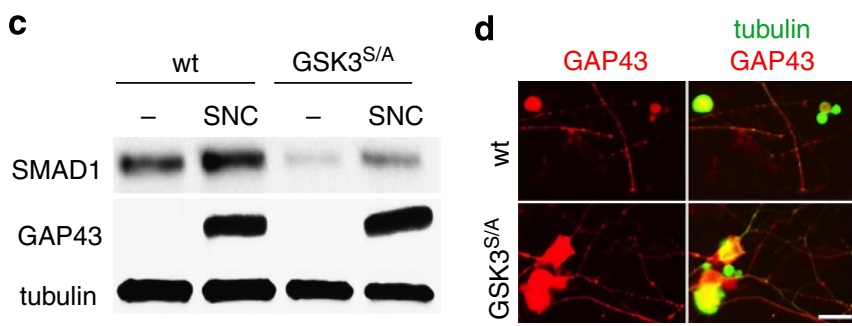

Figure 5 | Effect of sustained GSK3 activity on gene expression. (a,b) Quantitative real-time PCR of SPRR1a (a) and GAP43 (b) expression relative to GAPDH expression in adult DRG from either wild-type (wt) or $\mathrm{GSK}^{\mathrm{S} / \mathrm{A}}$ mice prior to sciatic nerve crush (-) or 5 days thereafter (SNC). Values represent means from four different animals. Error bars represent the standard error of the mean. Statistical significance was determined with ANOVA and the post-hoc Holm Sidac test. Measured treatment effects: ${ }^{\star \star} P \leq 0.001$. (c) Western-blot analysis showing SMAD1 and GAP43 expression prior to SNC ( - ) and 5 days after SNC in wt and $\mathrm{GSK}^{\mathrm{S} / \mathrm{A}}$ mice. Overall expression of SMAD1 is strongly reduced in GSK3 ${ }^{\mathrm{S} / \mathrm{A}}$ mice compared with wt animals, although both genotypes show an induction of SMAD1 following SNC. GAP43-protein levels are elevated similarly after SNC in both genotypes as compared with uninjured DRG. Tubulin served as loading control. (d) Immunocytochemical staining of dissociated adult DRG neurons (detected by $\beta$ III tubulin; green) verifies similar GAP43-protein expression (red) in GSK3 ${ }^{S / A}$ mice compared with wt controls after 3 days in culture, scale bar, $20 \mu \mathrm{m}$.

(refs 41,42) and occurring throughout the whole neuron. In contrast, mode I phosphorylation of MAP1B is concentrated in the distal part of the axon ${ }^{43}$ and switches microtubules into a dynamic state by promoting tyrosination of $\alpha$-tubulin ${ }^{40,44}$. Active GSK3 has been described to mediate this mode I phosphorylation at the two non-primed sites Serine 1,260 and Threonine 1,265 (refs 14,22,36). Decreased MAP1B mode I phosphorylation evoked by the application of GSK3 inhibitors reportedly reduces axon growth of embryonic chick DRG explants ${ }^{13}$ and dissociated cultures 6,39 . Moreover, GSK3-mediated phosphorylation of MAP1B compromises the detyrosination of microtubules, thereby keeping microtubules in a dynamically unstable state. This renders them more sensitive to nocodazole depolymerization, but also facilitates axon growth in embryonic tissue $^{13,16}$. As demonstrated by the current study, non-primed MAP1B phosphorylation by GSK3 is increased in adult neurons of GSK3 ${ }^{\mathrm{S} / \mathrm{A}}$ mice, and low concentrations of nocodazole specifically abolish the axon growth-promoting effect in GSK3 ${ }^{\mathrm{S} /}$ A, while wt neurons were not affected (Fig. 4b). Therefore, these data support the notion that GSK3 ${ }^{\text {S/A }}$ promotes axon growth, at least partially, via pMAP1B-dependent modulation of microtubules dynamics. Further investigations are required to address this possibility directly.

Beside the modulation of growth cone dynamics, constitutive GSK3 activity could affect the expression of regenerationassociated genes, thereby elevating the intrinsic growth state of the DRG neurons. GSK3 is known to interact with several transcription factors involved in axon growth ${ }^{45-47}$.

Albeit the underlying mechanism, maintaining GSK3 activity in GSK3 ${ }^{\text {S/A }}$ mice significantly accelerated axon regeneration in the injured sciatic nerve in vivo compared with wt mice. In $\mathrm{GSK}^{\mathrm{S} / \mathrm{A}}$ mice the number of axons at $3 \mathrm{~mm}$ beyond the lesion site was comparable to the number found at $1.5 \mathrm{~mm}$ in wt animals 3 days after injury, and re-established neuromuscular junctions in the EHL were only detected in GSK3 ${ }^{\mathrm{S} / \mathrm{A}}$ mice after 7 days. Consistent with the increased axonal growth observed in cell culture these data demonstrate that GSK $3^{\text {S/A }}$ markedly promotes axonal regeneration in vivo and accelerates functional recovery, as indicated by sensory and motor behavioural tests of GSK3 ${ }^{\text {S/A }}$ mice, compared with wt animals.

Therefore, therapies specifically preventing GSK3 phosphorylation without affecting PI3K activity, for instance by drugs or gene therapy, may constitute useful treatments to improve peripheral nerve regeneration. Future experiments will reveal whether the beneficial effects of constitutively active GSK3 seen in the current study are also applicable to CNS regeneration.

\section{Methods}

Surgical procedures. All animal protocols were approved by the local authorities and adhered to the respective animal care guidelines (Regierungspräsidium Recklinghausen). SNC was performed as described previously ${ }^{48}$. In brief, male and female adult (8-12 weeks) wt, homo and heterozygous GSK $3 \alpha^{\mathrm{S} 21 \mathrm{~A}} / \mathrm{GSK} 3 \beta^{\mathrm{S} 9 \mathrm{~A}}$ mice were maintained on a 12-h light/dark cycle with ad libitum access to food and water. Animals were housed under the same conditions for at least 10 days prior to use in experiments. Animals were anesthetized by intraperitoneal injections of ketamine (60-80 $\mathrm{mg} \mathrm{kg}^{-1}$, Pfizer, New York, NY, USA) and xylazine (10-15 $\mathrm{mg} \mathrm{kg}^{-1}$, Bayer, Leverkusen, Germany). A skin incision of about $10 \mathrm{~mm}$ was made over the gluteal region exposing the right sciatic nerve from the sciatic notch to the point of trifurcation. The ischiocrural musculature was carefully spread minimizing tissue damage. The crush injury was performed proximal to the tibial and peroneal divisions for $30 \mathrm{~s}$ using a Dumont \#5 forceps (Hermle, Tuttlingen, Germany) and marked with carbon (Sigma). The skin was sutured afterwards. The left sciatic nerve was left intact and served as untreated control, as indicated.

DRG neuron cultures and immunocytochemical staining procedure. DRG neurons were isolated from wt and homo and heterozygous GSK3 $\alpha^{\mathrm{S} 21 \mathrm{~A}} / \mathrm{GSK} 3 \beta^{\mathrm{S} 9 \mathrm{~A}}$ as described previously ${ }^{49}$. DRG (T8-L6) were harvested, incubated in $0.25 \%$ trypsin/EDTA (GE Healthcare, Chalfont St Giles, UK) and $0.3 \%$ collagenase type IA (Sigma, St Louis, MO, USA) in DMEM (Life Technologies, Carlsbad, CA, USA) at $37^{\circ} \mathrm{C}$ and $5 \% \mathrm{CO}_{2}$ for $45 \mathrm{~min}$ and mechanically dissociated. Cells were resuspended in DMEM containing 10\% foetal bovine serum (GE Healthcare) penicillin/streptomycin ( $500 \mathrm{U} \mathrm{ml}^{-1}$; Merck Millipore, Billerica, MA, USA) and 5 -fluoro-2'-desoxyuridine (100 nM; Sigma). Cells were cultured on poly-D-lysine $\left(0.1 \mathrm{mg} \mathrm{ml}^{-1}\right.$, molecular weight $<300,000 \mathrm{kDa}$; Sigma $)$ and laminin $\left(20 \mu \mathrm{g} \mathrm{ml}^{-1}\right.$, Sigma) coated 96 well plates (Nunc, Germany) at $37^{\circ} \mathrm{C}$ and $5 \% \mathrm{CO}_{2}$.

To test the effect of sustained GSK3 activity on axonal growth, dissociated DRG neurons from wt and GSK3 ${ }^{\mathrm{S} / \mathrm{A}}$ mice were cultured for $48 \mathrm{~h}$. Cells were then fixed with $4 \%$ paraformaldehyde (PFA; Sigma) and immunocytochemically stained with antibodies against $200 \mathrm{kDa}$ neurofilament heavy chain (1:2,000; ab8135, Abcam, Cambridge, UK) and $\beta$ III-tubulin (1:2,000; Covance, Princeton, NJ, USA). Imaging and quantification of total axon length and neuron numbers per well were automatically performed with the Pathway 855 microscope system (BD, Franklin Lakes, NJ, USA) and Attovision software, avoiding bias of quantification. Average axon length per neuron and neuron counts per group were normalized to control groups as indicated. Data are presented as the mean \pm s.e.m. of six replicate wells from three separate experiments. Significances of intergroup differences were evaluated using either one- or two-way analysis of variance (ANOVA) followed by Holm-Sidak post-hoc test.

To assess the effect of GSK3 $3^{\mathrm{S} / \mathrm{A}}$ on transcription-dependent axon growth as shown by Saijilafu et al. ${ }^{8}$, a replating method was employed as previously described. To this end DRG neurons were cultured for 3 days and then resuspended and replated on poly-D-lysine and laminin-coated culture dishes. After $20 \mathrm{~h}$ cells were fixed and immunohistochemically stained. Axonal growth was evaluated as described above.

To characterize the effect of various signalling pathways upon GSK3 phosphorylation in vitro, the following signalling pathway inhibitors were added to dissociated DRG cultures: Janus kinase 2 inhibitor AG490 (5 $\mu \mathrm{M}$, Merck Millipore), PI3k inhibitor LY294002 (1 $\mu \mathrm{M}$, Sigma), mTOR inhibitor rapamycin (10 nM, LCLaboratories, Woburn, MA, USA), mitogen-activated protein kinase inhibitors PD98059 and U0126 (both $5 \mu \mathrm{M}$, Merck Millipore) and GSK3 inhibitors Lithium 
(3 mM, Sigma) as well as SB415286 (5 $\mu \mathrm{M}$, Sigma). Cells were cultured for either $2 \mathrm{~h}$ or 3 days prior to fixation with 4\% PFA and immunocytochemically stained with antibodies against phospho-S9-GSK3 $\beta$ (1:500; Cell Signaling, Danvers, MA USA), phospho-S21-GSK3 $\alpha$ (1:500, Tebu-bio, Le-Perray-en-Yvelines, France), BIII-tubulin (1:2,000; Covance), GAP43 (1:500, Invitrogen, Carlsbad, CA, USA) and pMAP1B (1:500; Thr1265; Thermo Scientific, Waltham, MA, USA). Secondary antibodies were anti-mouse immunoglobulin $\mathrm{G}(\mathrm{IgG})$ and anti-rabbit $\operatorname{IgG}$ antibodies conjugated to either Alexa Fluor 488 or Alexa Fluor 594 (all 1:1,000; Molecular Probes, Eugene, OR, USA). Stained DRG neurons were analyzed via fluorescence microscopy (Zeiss STX). Data are represented as normalized means \pm s.e.m. from three separate experiments.

Western-blot assays. DRG (L3, L4) from four mice per experimental group were dissected and combined in $100 \mu$ lysis buffer $(20 \mathrm{mM}$ Tris $\mathrm{HCl} \mathrm{pH} 7.5,10 \mathrm{mM} \mathrm{KCl}$, $250 \mathrm{mM}$ sucrose, $10 \mathrm{mM} \mathrm{NaF}, 1 \mathrm{mM}$ DTT, $0.1 \mathrm{mM} \mathrm{Na}_{3} \mathrm{VO}_{4}, 1 \%$ TritonX-100, $0.1 \%$ SDS and protease inhibitors; Merck Millipore). DRG were homogenized by sonification and centrifuged at $8,000 \mathrm{~g}$ for $10 \mathrm{~min}$ at $4{ }^{\circ} \mathrm{C}$. Proteins in DRG lysates were separated by $10 \%$ SDS-polyacrylamide gel electrophoresis according to standard protocols. Proteins were then transferred to nitrocellulose membranes (Bio-Rad, Hercules, CA, USA). Blots were blocked either in $5 \%$ dried milk or $5 \%$ bovine serum albumin (Sigma) and incubated with either a monoclonal antibody against
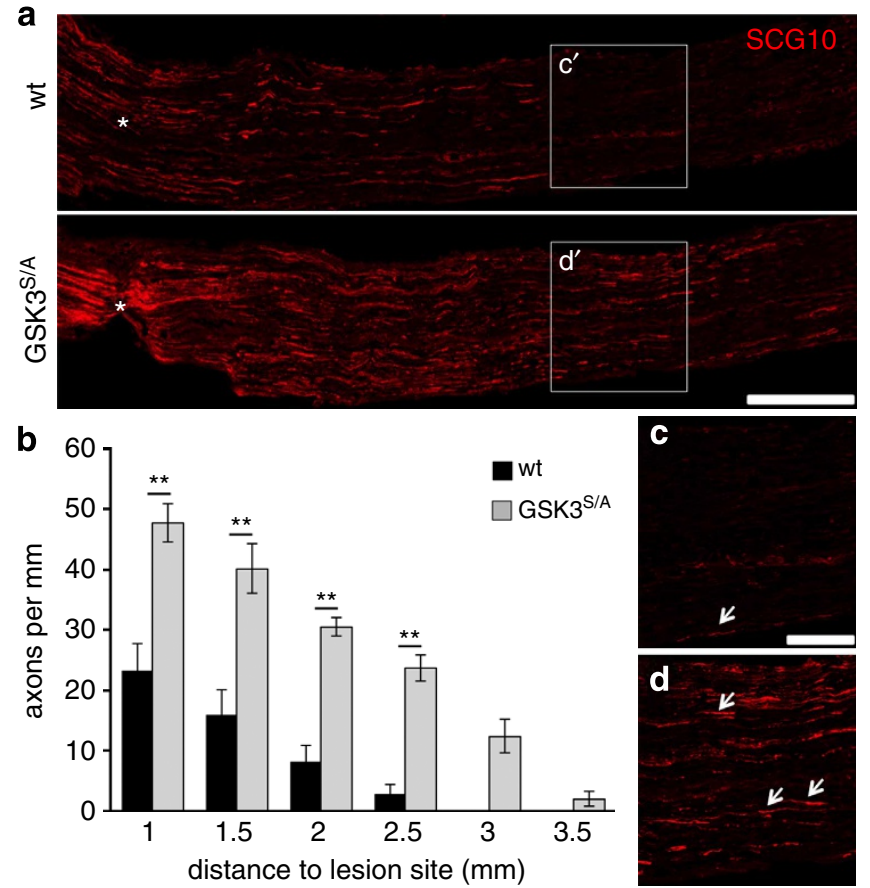

e

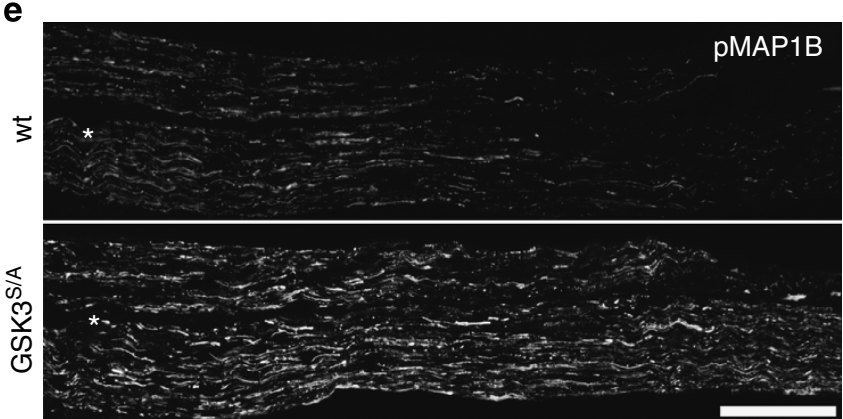

f wt GSK3 $^{\text {S/A }}$

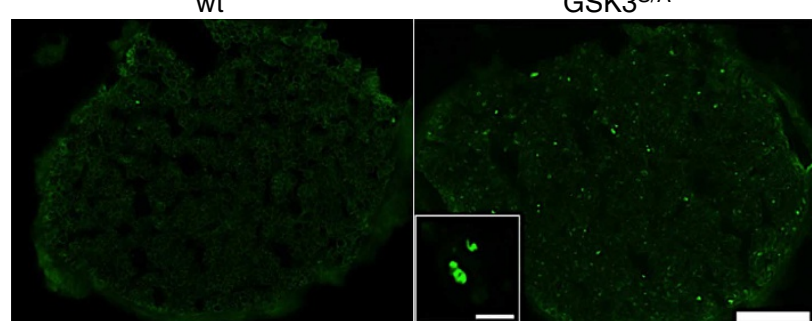

$\beta$-actin (1:7,500; Sigma), $\beta$ III-tubulin (1:2,000; Covance), SMAD1 (1:1,000; Cell Signaling), phospho-S21-GSK3 $\alpha$ (1:2,000; Cell Signaling), phospho-S9-GSK3 $\beta$ (1:2,000; Cell Signaling), total GSK3 $\alpha /$ GSK3 $\beta$ (1:500, Cell Signaling) or polyclonal antibodies against GAP43 (1:1,000; Invitrogen) at $4{ }^{\circ} \mathrm{C}$ overnight.

All antibodies except phospho-S21-GSK3 $\alpha$ were visualized with anti-rabbit or anti-mouse immunoglobulin $\mathrm{G}$ (IgG) secondary antibodies conjugated to horseradish peroxidase (1:80,000; Sigma). Phospho-S21-GSK3 $\alpha$ antibody was recognized with a horseradish peroxidase-conjugated anti-rabbit antibody (1:4.000; Cell Signaling). Antigen-antibody complexes were visualized using enhanced chemiluminescence substrate (Bio-Rad, Hercules, CA, USA). Western-blots were repeated at least twice to verify results.

Immunohistochemistry. Animals were killed by cervical dislocation either immediately, or 3 or 5 days after SNC. L3 and L4 DRG and sciatic nerves were isolated, post fixed for $6 \mathrm{~h}$, transferred to $30 \%$ sucrose overnight $\left(4^{\circ} \mathrm{C}\right)$ and embedded in Tissue-Tek (Sakura, Netherlands, Leiden). Longitudinal and crosssections of sciatic nerves and DRG were cut on a cryostat (Leica, Wetzlar, Germany), thaw-mounted onto coated glass slides (Superfrost plus, Fisher, Pittsburgh, PA, USA) and stored at $-20^{\circ} \mathrm{C}$ until further use. Monoclonal antibodies against $\beta$ III-tubulin (1:2,000; Convance), phospho-S21-GSK3 $\alpha$ (1:500; Tebu-bio, Offenbach, Germany) and phospho-S9-GSK3 $\beta$ (1:1,000; Cell Signaling) were used. Secondary antibodies included anti-mouse IgG and anti-rabbit IgG antibodies conjugated to either Alexa Fluor 488 or Alexa Fluor 594 (all 1:1,000; Invitrogen). Sections were embedded in Mowiol and analyzed using fluorescent microscopy. At least four animals per group were evaluated.

To analyze re-establishment of neuromuscular junctions, mice were killed at 3 and 7 days after SNC. The EHL muscle was dissected and post fixed in PFA for $1 \mathrm{~h}$. Afterwards muscles were permeabilized in $2 \%$ TritonX in PBS overnight. Axons where labelled with an antibody against neurofilament (1:2,000; Abcam). Synapses were visualized by incubation with $\alpha$-bungarotoxin conjugated to Alexa594 (1:1,000; Invitrogen) in PBS-Tween 20 for $1 \mathrm{~h}$.

RNA isolation and quantitative real-time PCR. For quantitative real-time PCR, DRG (L3, L4) from four mice per group were dissected and combined. Total RNA was isolated using the RNeasy mini kit (Qiagen, Hilden, Germany) according to the manufacturer's protocol. Total RNA (40 ng) was reverse transcribed using the Superscript II reverse transcriptase kit (Invitrogen). GAP43, SPRR1a and GAPDH expression was quantified using SYBR Green PCR Master Mix (Applied Biosystems, Foster City, CA, USA) and QuantiTect primers (MmGAP43_1_SG,

Mm_Gapdh_3_SG, Mm_Sprrla_2_SG; QuantiTect Primer Assay, Qiagen) on an Applied Biosystems 7,500 real-time PCR system (Life Technologies) using 50 amplification cycles according to the manufacturer's protocol. All reactions were performed in duplicate, and four independent samples from different mice were analyzed per experimental group. The specificity of the PCR product was verified with the dissociation curve analysis feature.

Quantification of regenerating axons in the sciatic nerve. Longitudinal cryosections $(14 \mu \mathrm{m})$ of sciatic nerves were immunohistochemically stained with an antibody against the regeneration-associated protein SCG10 (Novus Biologicals, Cambridge, UK) ${ }^{50}$ or against pMAP1B (Thermo Scientific). SCG10-positive axons

Figure 6 | GSK3 ${ }^{\text {S/A }}$ facilitates axon regeneration in the injured sciatic nerve. (a) Longitudinal sections of sciatic nerves from wild-type (wt) and GSK3 double knock-in mice (GSK3 ${ }^{S / A}$ ) were stained with a SCG10 antibody 3 days after injury. Scale bar, $500 \mu \mathrm{m}$. Asterisks indicate the crush site. (b) Quantification of SCG10 stained axons regenerating 1, 1.5, 2, 2.5, 3 and $3.5 \mathrm{~mm}$ beyond the lesion site 3 days after SNC. Five to eight animals were analyzed per treatment group. At least six sections per animal were analyzed. Data demonstrate significantly more regenerating axons at longer distances in the lesioned sciatic nerve of $\mathrm{GSK}^{\mathrm{S}} / \mathrm{A}$ compared with wt animals. The error bars represent the standard error of the mean. Statistical significance was determined with ANOVA and the post-hoc Holm Sidac test. Treatment effects: ${ }^{\star \star} P \leq 0.001$. (c,d) Higher magnification of sciatic nerve sections from wt and GSK3 $3 / \mathrm{A}$ animals as depicted in a approximately $2 \mathrm{~mm}$ distal from the crush site. Arrows indicate single regenerating axons. Scale bar, $160 \mu \mathrm{m}$. (e) Longitudinal sections of sciatic nerves from wt and $\mathrm{GSK}^{\mathrm{S}}{ }^{\mathrm{A}} \mathrm{A}$ were stained with a phospho-MAP1B (pMAP1B) antibody 3 days after sciatic nerve crush (SNC). Levels of PMAP1B are increased in regenerating GSK3 ${ }^{S / A}$ axons. Scale bar, $500 \mu \mathrm{m}$. Asterisks indicate the crush site. (f) Cross-sections of sciatic nerves from wt and GSK3 $3 / \mathrm{A} 3 \mathrm{~mm}$ distal to the lesion site were stained with a neurofilament antibody 3 days after sciatic nerve crush. Single axons are shown at larger magnification in the inset. Scale bar (whole nerve), $100 \mu \mathrm{m}$; scale bar (inset), $5 \mu \mathrm{m}$. 
a

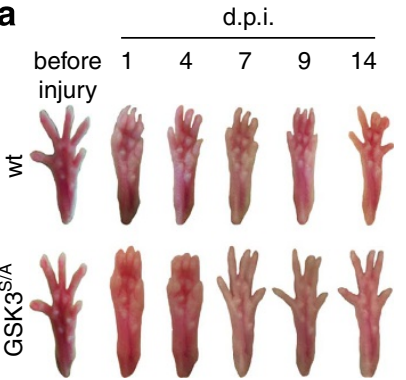

C

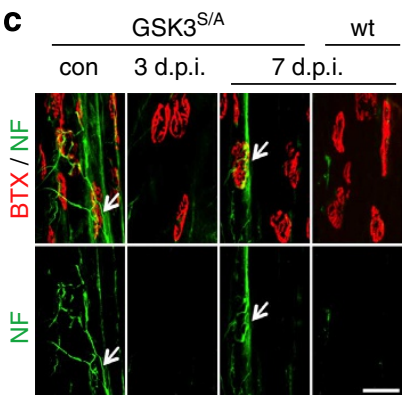

\section{b}

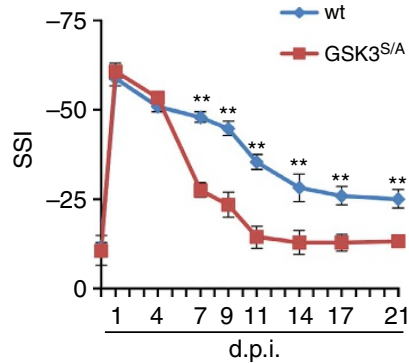

d

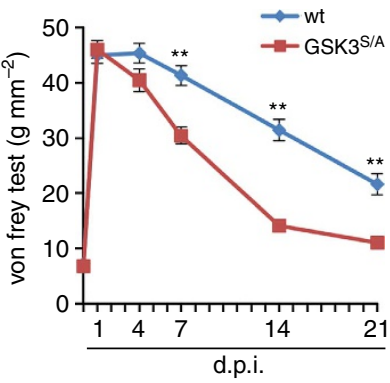

Figure 7 | GSK3 ${ }^{\text {S/A }}$ accelerates functional recovery upon sciatic nerve crush. (a) Representative pictures of the right hind paws of wild-type (wt) and GSK3 knock-in mice (GSK3 ${ }^{\mathrm{S} / \mathrm{A}}$ ) prior to and at 1, 4, 7, 9 and 14 days post sciatic nerve crush (d.p.i.), demonstrating the improved functional recovery in $\mathrm{GSK} 3^{\mathrm{S} / \mathrm{A}}$. (b) Quantification of functional motor recovery determined by the static sciatic index (SSI) of wt and GSK3 ${ }^{S / A}$ at various days post SNC. Treatment effects: ${ }^{\star \star} P \leq 0.001$. (c) $\alpha$-bungarowtoxin (BTX) and neurofilament staining of extensor hallucis longus wholemounts from wt and GSK3 ${ }^{\text {S/A }}$ without injury, 3 and 7 days after SNC. White arrows indicate synapses. Re-formation of neuromuscular junctions are seen in GSK3 ${ }^{S / A}$ at 7 d.p.i. (d) Sensory functional recovery determined by the von Frey test of wt and GSK3 ${ }^{S / A}$ at various days after injury. Treatment effects: ${ }^{\star \star} P \leq 0.001$.

were quantified at $1,1.5,2,2.5,3,3.5 \mathrm{~mm}$ beyond the carbon-labelled injury site, as described previously ${ }^{3}$. Statistical significances of intergroup differences were evaluated using a one-way ANOVA followed by the Holm-Sidak post-hoc test. Each experimental group included at least six sections from five different mice.

Static sciatic index. Functional motor recovery of $15 \mathrm{GSK} 3^{\mathrm{S} / \mathrm{A}}$ and $13 \mathrm{wt}$ mice after SNC was determined by calculating the SSI as described previously ${ }^{24,51}$. Mice were lifted from the ground to photograph the left and right hind paws, respectively. Toe spreading on the contra- (C, left) and ipsilateral (I, right) sides of the SNC was assessed in wt and transgenic GSK3 knock-in mice at 0, 1, 4, 7, 9, 11, 14,17 and 21 days after SNC, by measuring the paw length (PL) and the distance between the first and the fifth toe (FF). The SSI was calculated based on the previously described formula ${ }^{24}$ : SSI $=101.3($ (IFF-CFF)/CFF) - 54.03((IPL-CPL)/ CPL) -9.5 .

Data are represented as mean \pm s.e.m. from 13-15 animals per experimental group. Statistical significances of intergroup differences were evaluated using a twoway ANOVA followed by the Holm-Sidak post-hoc test.

Von frey test. Functional sensory recovery after SNC was determined with the von Frey filament test as described previously ${ }^{25}$ at $0,1,4,7,14$ and 21 days after SNC in $15 \mathrm{GSK}^{\mathrm{S} / \mathrm{A}}$ and $13 \mathrm{wt}$ mice. The test was performed at the same time of the day and by the same experimenter.

To this end, mice were placed on an elevated metal grid (grid size: $2 \mathrm{~mm}$ ) and allowed to acclimate for $15 \mathrm{~min}$ before testing. Then, responses of the ipsilateral hind paw to a range of innocuous von Frey filaments (Muromachi Kikai Co., LTD, Tokyo, Japan) were considered, starting with the smallest filament and increasing filament size until a positive response was initiated, which is indicated by a sharp withdrawal of the paw. Statistical significances of intergroup differences were evaluated using a two-way ANOVA followed by the Holm-Sidak post-hoc test.

\section{References}

1. Witzel, C., Rohde, C. \& Brushart, T. M. Pathway sampling by regenerating peripheral axons. J. Comp. Neurol. 485, 183-190 (2005).

2. Nguyen, Q. T., Sanes, J. R. \& Lichtman, J. W. Pre-existing pathways promote precise projection patterns. Nat. Neurosci. 5, 861-867 (2002).

3. Ma, C. H. et al. Accelerating axonal growth promotes motor recovery after peripheral nerve injury in mice. J. Clin. Invest. 121, 4332-4347 (2011).

4. Zhou, F. Q., Zhou, J., Dedhar, S., Wu, Y. H. \& Snider, W. D. NGF-induced axon growth is mediated by localized inactivation of GSK-3beta and functions of the microtubule plus end binding protein APC. Neuron 42, 897-912 (2004).

5. Dill, J., Wang, H., Zhou, F. \& Li, S. Inactivation of glycogen synthase kinase 3 promotes axonal growth and recovery in the CNS. J. Neurosci. 28, 8914-8928 (2008).

6. Alabed, Y. Z., Pool, M., Ong Tone, S., Sutherland, C. \& Fournier, A. E. GSK3 beta regulates myelin-dependent axon outgrowth inhibition through CRMP4. J. Neurosci. 30, 5635-5643 (2010).

7. Hur, E. M. \& Zhou, F. Q. GSK3 signalling in neural development. Nat. Rev. Neurosci. 11, 539-551 (2010).

8. Saijilafu et al. PI3K-GSK3 signalling regulates mammalian axon regeneration by inducing the expression of Smad1. Nat. Commun. 4, 2690 (2013).

9. Liu, C. M., Hur, E. M. \& Zhou, F. Q. Coordinating gene expression and axon assembly to control axon growth: potential role of gsk3 signaling. Front. Mol. Neurosci. 5, 3 (2012).

10. Meijer, L., Flajolet, M. \& Greengard, P. Pharmacological inhibitors of glycogen synthase kinase 3. Trends Pharmacol. Sci. 25, 471-480 (2004).

11. Sutherland, C. \& Cohen, P. The alpha-isoform of glycogen synthase kinase-3 from rabbit skeletal muscle is inactivated by p70 S6 kinase or MAP kinaseactivated protein kinase-1 in vitro. FEBS Lett. 338, 37-42 (1994).

12. Sutherland, C., Leighton, I. A. \& Cohen, P. Inactivation of glycogen synthase kinase- 3 beta by phosphorylation: new kinase connections in insulin and growth-factor signalling. Biochem. J. 296(Pt 1): 15-19 (1993).

13. Owen, R. \& Gordon-Weeks, P. R. Inhibition of glycogen synthase kinase 3beta in sensory neurons in culture alters filopodia dynamics and microtubule distribution in growth cones. Mol. Cell. Neurosci. 23, 626-637 (2003).

14. Lucas, F. R., Goold, R. G., Gordon-Weeks, P. R. \& Salinas, P. C. Inhibition of GSK-3beta leading to the loss of phosphorylated MAP-1B is an early event in axonal remodelling induced by WNT-7a or lithium. J. Cell. Sci. 111(Pt 10): 1351-1361 (1998).

15. Krylova, O. et al. WNT-3, expressed by motoneurons, regulates terminal arborization of neurotrophin-3-responsive spinal sensory neurons. Neuron 35, 1043-1056 (2002).

16. Goold, R. G., Owen, R. \& Gordon-Weeks, P. R. Glycogen synthase kinase 3beta phosphorylation of microtubule-associated protein $1 \mathrm{~B}$ regulates the stability of microtubules in growth cones. J. Cell. Sci. 112(Pt 19): 3373-3384 (1999).

17. McManus, E. J. et al. Role that phosphorylation of GSK3 plays in insulin and Wnt signalling defined by knockin analysis. EMBO J. 24, 1571-1583 (2005).

18. Rigaud, M. et al. Species and strain differences in rodent sciatic nerve anatomy: implications for studies of neuropathic pain. Pain 136, 188-201 (2008).

19. Chalecka-Franaszek, E. \& Chuang, D. M. Lithium activates the serine/threonine kinase Akt-1 and suppresses glutamate-induced inhibition of Akt-1 activity in neurons. Proc. Natl Acad. Sci. USA 96, 8745-8750 (1999).

20. Coghlan, M. P. et al. Selective small molecule inhibitors of glycogen synthase kinase-3 modulate glycogen metabolism and gene transcription. Chem. Biol. 7, 793-803 (2000).

21. Zhou, F. Q. \& Snider, W. D. Cell biology. GSK-3beta and microtubule assembly in axons. Science 308, 211-214 (2005).

22. Scales, T. M., Lin, S., Kraus, M., Goold, R. G. \& Gordon-Weeks, P. R. Nonprimed and DYRK1A-primed GSK3 beta-phosphorylation sites on MAP1B regulate microtubule dynamics in growing axons. J. Cell. Sci. 122, 2424-2435 (2009).

23. Hur, E. M. et al. GSK3 controls axon growth via CLASP-mediated regulation of growth cone microtubules. Genes Dev. 25, 1968-1981 (2011).

24. Baptista, A. F. et al. A new approach to assess function after sciatic nerve lesion in the mouse-adaptation of the sciatic static index. J. Neurosci. Methods 161, 259-264 (2007).

25. Gladman, S. J. et al. Improved outcome after peripheral nerve injury in mice with increased levels of endogenous omega-3 polyunsaturated fatty acids. J. Neurosci. 32, 563-571 (2012).

26. Quigley, H. A. \& Broman, A. T. The number of people with glaucoma worldwide in 2010 and 2020. Br. J. Ophthalmol. 90, 262-267 (2006).

27. Liu, K. et al. PTEN deletion enhances the regenerative ability of adult corticospinal neurons. Nat. Neurosci. 13, 1075-1081 (2010).

28. Park, K. K. et al. Promoting axon regeneration in the adult CNS by modulation of the PTEN/mTOR pathway. Science 322, 963-966 (2008).

29. Jones, D. M., Tucker, B. A., Rahimtula, M. \& Mearow, K. M. The synergistic effects of NGF and IGF-1 on neurite growth in adult sensory neurons: convergence on the PI 3-kinase signalling pathway. J. Neurochem. 86, 1116-1128 (2003). 
30. Fischer, D. \& Leibinger, M. Promoting optic nerve regeneration. Prog. Retin. Eye Res. 31, 688-701 (2012)

31. Cross, D. A., Alessi, D. R., Cohen, P., Andjelkovich, M. \& Hemmings, B. A. Inhibition of glycogen synthase kinase- 3 by insulin mediated by protein kinase B. Nature 378, 785-789 (1995).

32. Cohen, P. \& Frame, S. The renaissance of GSK3. Nature reviews. Mol. Cell Biol. 2, 769-776 (2001).

33. Knockaert, M., Greengard, P. \& Meijer, L. Pharmacological inhibitors of cyclindependent kinases. Trends Pharmacol. Sci. 23, 417-425 (2002).

34. Fischer, P. M. CDK versus GSK-3 inhibition: a purple haze no longer? Chem. Biol. 10, 1144-1146 (2003).

35. Nishino, Y. et al. Glycogen synthase kinase-3 inactivation is not required for ischemic preconditioning or postconditioning in the mouse. Circ. Res. 103, 307-314 (2008)

36. Trivedi, N., Marsh, P., Goold, R. G., Wood-Kaczmar, A. \& Gordon-Weeks, P. R. Glycogen synthase kinase-3beta phosphorylation of MAP1B at Ser1260 and Thr1265 is spatially restricted to growing axons. J. Cell. Sci. 118, 993-1005 (2005).

37. Zou, H., Ho, C., Wong, K. \& Tessier-Lavigne, M. Axotomy-induced Smad1 activation promotes axonal growth in adult sensory neurons. J. Neurosci. 29, $7116-7123$ (2009)

38. Zhou, F. Q. \& Snider, W. D. Intracellular control of developmental and regenerative axon growth. Philos. Trans. R Soc. Lond. B Biol. Sci. 361, 1575-1592 (2006).

39. Kim, W. Y. et al. Essential roles for GSK-3s and GSK-3-primed substrates in neurotrophin-induced and hippocampal axon growth. Neuron 52, 981-996 (2006).

40. Gonzalez-Billault, C., Avila, J. \& Caceres, A. Evidence for the role of MAP1B in axon formation. Mol. Biol. Cell 12, 2087-2098 (2001).

41. Avila, J., Ulloa, L., Diez-Guerra, J. \& Diaz-Nido, J. Role of phosphorylated MAPIB in neuritogenesis. Cell Biol. Int. 18, 309-314 (1994).

42. Diaz-Nido, J., Serrano, L., Mendez, E. \& Avila, J. A casein kinase II-related activity is involved in phosphorylation of microtubule-associated protein MAP1B during neuroblastoma cell differentiation. J. Cell Biol. 106, 2057-2065 (1988).

43. Ulloa, L., Diez-Guerra, F. J., Avila, J. \& Diaz-Nido, J. Localization of differentially phosphorylated isoforms of microtubule-associated protein $1 \mathrm{~B}$ in cultured rat hippocampal neurons. Neuroscience 61, 211-223 (1994).

44. Gonzalez-Billault, C. et al. Microtubule-associated protein 1B function during normal development, regeneration, and pathological conditions in the nervous system. J. Neurobiol. 58, 48-59 (2004).
45. Turenne, G. A. \& Price, B. D. Glycogen synthase kinase3 beta phosphorylates serine 33 of p53 and activates p53's transcriptional activity. BMC Cell Biol. 2, 12 (2001).

46. Watcharasit, P. et al. Glycogen synthase kinase-3beta (GSK3beta) binds to and promotes the actions of p53. J. Biol. Chem. 278, 48872-48879 (2003).

47. Watcharasit, P. et al. Direct, activating interaction between glycogen synthase kinase-3beta and p53 after DNA damage. Proc. Natl Acad. Sci. USA 99, 7951-7955 (2002).

48. Elfar, J. C., Jacobson, J. A., Puzas, J. E., Rosier, R. N. \& Zuscik, M. J. Erythropoietin accelerates functional recovery after peripheral nerve injury. J. Bone Joint. Surg. Am. 90, 1644-1653 (2008).

49. Scott, B. S. Adult mouse dorsal root ganglia neurons in cell culture. J. Neurobiol. 8, 417-427 (1977).

50. Shin, J. E. et al. SCG10 is a JNK target in the axonal degeneration pathway. Proc. Natl Acad. Sci. USA 109, E3696-E3705 (2012).

51. Bobinski, F. et al. Neuroprotective and neuroregenerative effects of lowintensity aerobic exercise on sciatic nerve crush injury in mice. Neuroscience 194, 337-348 (2011)

\section{Acknowledgements}

We are grateful to Professor Alessi for providing the GSK3 double knock-in mice. We thank Dr Heike Diekmann for helpful comments and suggestions and Marcel Kohlhaas, the University of Düsseldorf for technical support. This work was supported by the German Research Foundation (D.F.).

\section{Author contributions}

D.F. designed the project. P.G., M.L. and A.A. performed the experiments. D.F., P.G. and M.L. analyzed the data. D.F. supervised the research; D.F., P.G. and M.L. wrote the paper.

\section{Additional information}

Supplementary Information accompanies this paper at http://www.nature.com/ naturecommunications

Competing financial interests: The authors declare no competing financial interests.

Reprints and permission information is available online at http://npg.nature.com/ reprintsandpermissions/

How to cite this article: Gobrecht, P. et al. Sustained GSK3 activity markedly facilitates nerve regeneration. Nat. Commun. 5:4561 doi: 10.1038/ncomms5561 (2014). 